\title{
Valuations in fields of power series
}

\author{
Francisco Javier Herrera Govantes, Miguel Ángel Olalla Acosta \\ and José Luis Vicente Córdoba
}

\begin{abstract}
This paper deals with valuations of fields of formal meromorphic functions and their residue fields. We explicitly describe the residue fields of the monomial valuations. We also classify all the discrete rank one valuations of fields of power series in two and three variables, according to their residue fields. We prove that all our cases are possible and give explicit constructions.
\end{abstract}

\section{Introduction}

In this paper, we give a "classification" of certain valuations of $k\left(\left(X_{1}, \ldots, X_{n}\right)\right)$, where $k$ is an algebraically closed field, namely discrete valuations finite over $k \llbracket X_{1}, \ldots, X_{n} \rrbracket$ and having as center in it the maximal ideal.

In section 2, we construct explicitly the residue field of the discrete monomial valuations of any rank. Section 3 is devoted to list in detail all the discrete rank one valuations of $k((X)), k\left(\left(X_{1}, X_{2}\right)\right), k\left(\left(X_{1}, X_{2}, X_{3}\right)\right)$ (again with the condition on the center $)$. The case of $k((X))$ is trivial.

In the case of $k\left(\left(X_{1}, X_{2}\right)\right)$ we get that the residue field $\Delta_{v}$ of any such valuation $v$ is a pure transcendental extension $k(u)$ of $k$ generated by one element, and $v$ itself is in one of the following two cases:

1. either $v$ is monomial, or

2. $v$ is the composition of a finite number of blowing-ups and coordinate changes with a monomial valuation.

2000 Mathematics Subject Classification: Primary 12J20; Secondary 13A18.

Keywords: Valuation Theory, Power Series. 
In the case of $k\left(\left(X_{1}, X_{2}, X_{3}\right)\right)$, the situation is much more complicated. To compute the residue field and to give an explicit expression of $v$, we allow ourselves to perform sequences (maybe infinite) of blowing-ups and coordinate changes. The possible cases are the following:

1. $\Delta_{v}=k(u)$. In this case, $k \llbracket X_{1}, X_{2}, X_{3} \rrbracket$ can be embedded into a power series ring in two variables contained in the ring of the completion of $v$, and the extension to it of $v$ is monomial.

2. $\Delta_{v}$ has transcendence degree 2 over $k$, and the algebraic part may be non-trivial. This case includes the monomial valuations and the compositions of a finite sequence of blowing-ups with such a valuation.

3. $\Delta_{v}$ has transcendence degree 1 and the algebraic part may be finite or countably infinite. If $u$ is transcendental over $k$, every countably generated algebraic extension of $k(u)$ can be realized as the residue field $\Delta_{v}$ of the valuation $v$.

Section 4 is devoted to discrete rank two valuations of $k\left(\left(X_{1}, X_{2}\right)\right)$. The principles of the process of treatment are similar to the rank one case. The result is that any discrete rank two valuation of $k\left(\left(X_{1}, X_{2}\right)\right)$ is zerodimensional and:

1. either it's monomial,

2. or it can be reduced to a monomial one by a (possibly infinite) sequence of blowing-ups and coordinate changes.

\section{Monomial valuations}

Throughout all this paper, $k$ will be an algebraically closed field, $R=k \llbracket \mathbf{X} \rrbracket=$ $k \llbracket X_{1}, \ldots, X_{n} \rrbracket$ the formal power series ring in the variables $X_{i}$ and $K$ its quotient field.

Remark 1 (1) Every $f \in R$ will be written as $f=\sum_{A \in \mathbb{Z}_{0}^{n}} f_{A} \mathbf{X}^{A}$, where, if $A=\left(a_{1}, \ldots, a_{n}\right)$, then $\mathbf{X}^{A}$ means $X_{1}^{a_{1}} \cdots X_{n}^{a_{n}}$. We will also write

$$
\mathcal{E}(f)=\left\{A \in \mathbb{Z}_{0}^{n} \mid f_{A} \neq 0\right\}
$$

(2) We will freely speak about valuations of $R$, meaning the restriction to $R$ of a valuation of its quotient field. To abbreviate, the word "valuation" will replace the phrase "discrete $k$-valuation of $R$ centered at the maximal ideal $M=(\mathbf{X}) \cdot R "$. 
(3) We will use in every $\mathbb{Z}^{m}$ the lexicographic order, which will be denoted by $\leq_{\text {lex }}$. It is a monomial order in the sense that it is compatible with the additive group structure.

(4) Let $0<m \leq n$ be an integer and

$$
L=\left\{B_{1}, \ldots, B_{n}\right\} \subset \mathbb{Z}_{0}^{m} \backslash\{\mathbf{0}\}
$$

a system of generators of $\mathbb{Z}^{m}$. We associate to each monomial $\mathbf{X}^{A}$ in $R$ an element of $\mathbb{Z}_{0}^{m}$, which we call its $L$-degree, defined in the following way:

$$
\text { degree }_{L}\left(\mathbf{X}^{A}\right)=\sum_{i=1}^{n} a_{i} B_{i}, \quad A=\left(a_{1}, \ldots, a_{n}\right) .
$$

Lemma 1 Let $B=\left(b_{1}, \ldots, b_{m}\right) \in \mathbb{Z}_{0}^{m}$; then the set of monomials with $L$-degree equal $B$ is finite. Consequently, the homogeneous components of the $L$-graduation of $R$ are finite dimensional $k$-vector spaces.

Proof: It is enough to observe that, since all the $B_{i}$ are different from zero, any linear combination $B=\sum_{i=1}^{n} a_{i} B_{i}$ with $a_{i} \in \mathbb{Z}_{0}$ must have the coefficients bounded by the maximum of the $b_{j}$.

Definition 2 Let $0<m \leq n$ be an integer and

$$
L=\left\{B_{1}, \ldots, B_{n}\right\} \subset \mathbb{Z}_{0}^{m} \backslash\{\mathbf{0}\}
$$

a system of generators of $\mathbb{Z}^{m}$. Let $v: R \rightarrow \mathbb{Z}^{m} \cup\{\infty\}$ be the function which assigns $\infty$ to zero and

$$
v(f)=\min _{\text {lex }}\left\{\operatorname{degree}_{L}\left(\mathbf{X}^{A}\right) \mid A \in \mathcal{E}(f)\right\}
$$

to $0 \neq f \in R$. The extension of $v$ to $K$ is a valuation of $K / k$ whose value group is $\mathbb{Z}^{m}$, which will be called the monomial valuation associated to $L$.

Remark 2 Let $A_{1}, \ldots, A_{p} \in \mathbb{Z}^{n}$; the following conditions are equivalent:

a) The monomials $\left\{\mathbf{X}^{A_{1}}, \ldots, \mathbf{X}^{A_{p}}\right\}$ are algebraically independent over $k$ (they may have negative exponents).

b) $\left\{A_{1}, \ldots, A_{p}\right\}$ are $\mathbb{Z}$-linearly independent in $\mathbb{Z}^{n}$.

Proposition 3 Let $v$ be a valuation of $K / k$ of rank $m$. Let $B_{i}=v\left(X_{i}\right)$ for $i=1, \ldots, n$, and consider the set $L=\left\{B_{1}, \ldots, B_{n}\right\}$; we will further assume that $L$ is a system of generators of $\mathbb{Z}^{m}$. Let $v_{L}$ be the corresponding monomial valuation of $K / k$. Then, for every $f \in R$, one has $v(f) \geq_{\operatorname{lex}} v_{L}(f)$. 
Proof: Let us assume that $f \neq 0$ and let $v_{L}(f)=B$. Let $\mathbf{X}^{A}$ be a monomial with non-zero coefficient in $f$ of $L$-degree $B$ and consider the fraction $g / \mathbf{X}^{A}$. It is clear that $g / \mathbf{X}^{A} \in R\left[Y_{1}, \ldots, Y_{s}\right]$, where each $Y_{i}$ is a quotient of monomials in $\mathbf{X}$ of value zero. It is also clear that $R\left[Y_{1}, \ldots, Y_{s}\right] \subset R_{v}$, hence $v\left(g / \mathbf{X}^{A}\right) \geq_{\text {lex }} 0$, so $v(g) \geq_{\text {lex }} v\left(\mathbf{X}^{A}\right)=B$. This completes our proof.

Theorem 4 Let $v$ be a valuation of $K / k$ of rank $m, B_{i}=v\left(X_{i}\right)$ for $i=$ $1, \ldots, n$ and let us assume that $L=\left\{B_{1}, \ldots, B_{n}\right\}$ is a system of generators of $\mathbb{Z}^{m}$. Then the following conditions are equivalent:

1. $v$ is the monomial valuation associated to $L$.

2. The residue field $\Delta_{v}$ of $v$ is the extension of $k$ (pure transcendental if $n>m$ ) generated by the $n-m$ monomials (fractional in general) whose exponents constitute a basis of the solutions of the system $\sum_{i=1}^{n} y_{i} B_{i}=\mathbf{0}$, as an abelian group.

Proof: Here we are using the known fact (although not completely trivial) that the solutions in $\mathbb{Z}^{m}$ of a system of homogeneous linear equations is a free abelian group (c.f. [4]). Let us assume that $v$ is the monomial valuation associated to $L$ and let $K_{L}$ be the subfield of $K$ consisting of the quotients of $L$-forms of the same $L$-degree. The natural map

$$
\begin{aligned}
K_{L} & \longrightarrow \Delta_{v} \\
\frac{f_{B}}{g_{B}} & \longmapsto \frac{f_{B}}{g_{B}}+\mathcal{M}_{v}
\end{aligned}
$$

is injective, so we may assume $K_{L} \subset \Delta_{v}$. Let $f / g \in K$ be such that $v(f / g)=0$, that is, $f$ and $g$ are two power series such that $v(f)=v(g)=B$.

Let us write $f=f_{B}+f_{1}$ and $g=g_{B}+g_{1}$, where $f_{B}$ and $g_{B}$ are their $L$-initial forms respectively. Then,

$$
\frac{f}{g}-\frac{f_{B}}{g_{B}}=\frac{g_{B} f_{1}-f_{B} g_{1}}{g_{B} g},
$$

so $v\left(f / g-f_{B} / g_{B}\right)>0$ and

$$
\frac{f}{g}+\mathcal{M}_{v}=\frac{f_{B}}{g_{B}}+\mathcal{M}_{v}
$$

therefore $K_{L}=\Delta_{v}$.

Let $M$ be the submodule of $\mathbb{Z}^{m}$ consisting of the solutions of the system $\sum_{i=1}^{n} y_{i} B_{i}=\mathbf{0}$ and $\left\{A_{1}, \ldots, A_{n-m}\right\}$ be a base of $M$. Then one easily checks that

$$
K_{L}=k\left(\mathbf{X}^{A_{1}}, \ldots, \mathbf{X}^{A_{n-m}}\right)
$$


Now let us assume that the residue field of $v$ is $\Delta_{v}=k\left(\mathbf{X}^{A_{1}}, \ldots, \mathbf{X}^{A_{n-m}}\right)$, where $A_{1}, \ldots, A_{n-m}$ form a basis of the submodule $M$ of the solutions of the system $\sum_{i=1}^{n} y_{i} B_{i}=\mathbf{0}$. Let $v_{L}$ be the monomial valuation associated to $L$. By proposition 3 , in order to show that $v=v_{L}$ it is enough to prove that $v(f)=v_{L}(f)$ for every $L$-form $f$.

Assume the contrary and let $f$ be an $L$-form such that $v(f)>_{\text {lex }} v_{L}(f)$. Let us write $f=\sum_{i=1}^{s} \alpha_{i} \mathbf{X}^{C_{i}}$ where $\alpha_{i} \in k$ and the sum is extended to all the monomials of $L$-degree $v_{L}(f)$. For every $j=1, \ldots, n$ one has $v\left(f / \mathbf{X}^{C_{j}}\right)>0$, hence

$$
\frac{f}{\mathrm{X}^{C_{j}}}+\mathcal{M}_{v}=0+\mathcal{M}_{v}
$$

Since

$$
\frac{f}{\mathbf{X}^{C_{j}}}=\alpha_{j}+\sum_{i \neq j} \alpha_{i} \mathbf{X}^{C_{i}-C_{j}}
$$

one has $\sum_{i \neq j} \alpha_{i} \mathbf{X}^{C_{i}-C_{j}}+\mathcal{M}_{v}=-a_{j}+\mathcal{M}_{v}$. This implies that $\sum_{i \neq j} \alpha_{i} \mathbf{X}^{C_{i}-C_{j}}+$ $\mathcal{M}_{v}$ belongs to $k$, which is not possible since all the $\mathbf{X}^{C_{j}-C_{i}}$ are monomials in the $A_{l}$ and these are algebraically independent over $k$.

\section{Discrete rank one valuations in low dimension}

We now consider other discrete valuations of fields of power series. It is easy to make a complete list of all the discrete rank one valuations of $k\left(\left(X_{1}\right)\right) / k$ and $k\left(\left(X_{1}, X_{2}\right)\right) / k$, and we do it in a few considerations. However, the case of three variables is more difficult. We will explicitly describe all the discrete rank one valuations of $k\left(\left(X_{1}, X_{2}, X_{3}\right)\right) / k$ because it is the most difficult one.

Remark 3 (1) As before, let $R=k \llbracket \mathbf{X} \rrbracket=k \llbracket X_{1}, \ldots, X_{n} \rrbracket, n>1$, and $K$ be its quotient field. We fix a discrete rank one valuation

$$
v: K=k\left(\left(X_{1}, \ldots, X_{n}\right)\right) \longrightarrow \mathbb{Z} \cup \infty,
$$

centered at the maximal ideal of $R$ and we assume that the value group is $\mathbb{Z}$. We will denote, as usual, by $R_{v}, \mathcal{M}_{v}$ and $\Delta_{v}$ the ring, the ideal and the residue field of $v$.

(2) We will consider the completion $\widehat{v}$ of $v$, together with its ring $\widehat{R}_{\widehat{v}}$ and the quotient field $\widehat{K}_{\widehat{v}}$ of $\widehat{R}_{\widehat{v}}$. We will fix a datum, which will play a key role in our study, namely a section $\sigma: \Delta_{v} \rightarrow \widehat{R}_{\widehat{v}}$ of the natural homomorphism $\widehat{R}_{\widehat{v}} \rightarrow \Delta_{v}$, which exists by the Cohen structure theorem. We will always identify $\Delta_{v}$ with its image in $\widehat{R}_{\widehat{v}}$ by $\sigma$, so we will assume from now on that $\Delta_{v} \subset \widehat{R}_{\widehat{v}}$.

(3) Remark that, if $t \in \widehat{R}_{\widehat{v}}$ is an element of value 1 , then $t$ is formally independent over $\Delta_{v}$ and $\widehat{R}_{\widehat{v}}=\Delta_{v} \llbracket t \rrbracket$. 
Remark 4 Let us assume that $n=1$; then the usual order function $\nu_{X_{1}}$ is a discrete rank one valuation whose ring is $k \llbracket X_{1} \rrbracket$ and its residue field is $k$. Every other discrete valuation $v$ of $k\left(\left(X_{1}\right)\right) / k$ such that $R_{v} \supset k \llbracket X_{1} \rrbracket$ and $\mathcal{M}_{v} \cap k \llbracket X_{1} \rrbracket=\left(X_{1}\right)$ must coincide with $\nu_{X_{1}}$. Thus the only non trivial valuation of rank $m=1$ of $k\left(\left(X_{1}\right)\right) / k$ is the usual order function.

Remark 5 Let us assume that $v$ is a discrete rank one valuation of $K / k$, where $K=k\left(\left(X_{1}, \ldots, X_{n}\right)\right)$, and refer to the above notations.

(1) Let $z_{1}, z_{2} \in \widehat{\mathcal{M}}_{\widehat{v}}$ and $L$ a subfield of $\widehat{R}_{\widehat{v}}$ containing $k$, i.e., such that $k \subset L \subset \Delta_{v}$. Let us assume that $\widehat{v}\left(z_{2}\right)>\widehat{v}\left(z_{1}\right)>0$; then, the natural map $k \llbracket z_{1}, z_{2} / z_{1} \rrbracket \rightarrow \widehat{R}_{\widehat{v}}$ is injective, as we will see in a moment (cf. (3)).

We will assume that $k \llbracket z_{1}, z_{2} / z_{1} \rrbracket \rightarrow \widehat{R}_{\widehat{v}}$ is injective. Therefore, $z_{2} / z_{1} \in \widehat{\mathcal{M}}_{\widehat{v}}$, every power series $f\left(z_{1}, z_{2} / z_{1}\right) \in L \llbracket z_{1}, z_{2} / z_{1} \rrbracket$ makes sense in $\widehat{R}_{\widehat{v}}, L \llbracket z_{1}, z_{2} / z_{1} \rrbracket$ $\subset \widehat{R}_{\widehat{v}}$ and $L \llbracket z_{1}, z_{2} \rrbracket \subset L \llbracket z_{1}, z_{2} / z_{1} \rrbracket$. This $L \llbracket z_{1}, z_{2} / z_{1} \rrbracket$ is called the blowing-up of $L \llbracket z_{1}, z_{2} \rrbracket$. If $\widehat{v}\left(z_{2}\right)=q \widehat{v}\left(z_{1}\right)+r$ is the euclidean division and $r>0$, then $q$ repetitions of the blowing-up dividing by $z_{1}$ give us the ring $L \llbracket z_{1}, z_{2} / z_{1}^{q} \rrbracket \supset$ $L \llbracket z_{1}, z_{2} \rrbracket$ in which $\widehat{v}\left(z_{2} / z_{1}^{q}\right)=r$. If the remainder is zero, we usually take $q-1$ blowing-ups instead of $q$ just to equate the values. The pair $\left(z_{1}, z_{2} / z_{1}^{q}\right)$ is monomial, birrational with respect to $\left(z_{1}, z_{2}\right)$, i.e., each one is a monomial in the other, possibly with negative exponents. Equivalently, the vectors of the exponents $\{(1,0),(-q, 1)\}$ form a basis of $\mathbb{Z}^{2}$.

(2) Let $d=\operatorname{gcd}\left(v\left(X_{1}\right), \ldots, v\left(X_{n}\right)\right)$; then a finite sequence of bowing-ups (describing the Euclid's algorithms to compute the greatest common divisors in the sense of (1)) will produce a vector $\mathbf{z}=\left(z_{1}, \ldots, z_{n}\right)$ of elements in $\widehat{\mathcal{M}}_{\widehat{v}}$ such that $\widehat{v}\left(z_{i}\right)=d, \forall i=1, \ldots, n$. The important point here is that $k \llbracket \mathbf{z} \rrbracket \supset k \llbracket \mathbf{X} \rrbracket$ and the vectors $\mathbf{z}$ and $\mathbf{X}$ are monomial birrational.

This is obviously true for every starting vector $\mathbf{x}=\left(x_{1}, \ldots, x_{n}\right)$ with components in $\widehat{\mathcal{M}}_{\widehat{v}}$, either variables (i.e., formally independent) or not, over any starting field. This process will be called reduction of a vector to the minimum value.

(3) In the process of reduction of a vector to the minimum value, a crucial point is that, if the starting vector has two components formally independent over the ground field $L$, then the two components of the final vector are also formally independent over $L$. To see this, it is enough to prove that, if $z_{1}, z_{2}$ are formally independent over $L$, then $z_{1}, z_{2} / z_{1}$ are also formally independent over $L$. Reasoning by contradiction, let us assume that $z_{1}, z_{2} / z_{1}$ are not formally independent over $L$ and let $f\left(Z_{1}, Z_{2}^{\prime}\right) \in L \llbracket Z_{1}, Z_{2}^{\prime} \rrbracket$ be such that $f\left(z_{1}, z_{2} / z_{1}\right)=0$; then $f$ cannot be a unit. 
By the Weierstrass preparation theorem, there is a unit $u\left(Z_{1}, Z_{2}^{\prime}\right)$ and a non-unit Weierstrass polynomial $g\left(Z_{1}, Z_{2}^{\prime}\right)$ in $Z_{2}^{\prime}$ such that $f\left(Z_{1}, Z_{2}^{\prime}\right)=$ $Z_{1}^{r} u\left(Z_{1}, Z_{2}^{\prime}\right) g\left(Z_{1}, Z_{2}^{\prime}\right)$. Thus

$$
0=f\left(z_{1}, z_{2} / z_{1}\right)=z_{1}^{r} u\left(z_{1}, z_{2} / z_{1}\right) g\left(z_{1}, z_{2} / z_{1}\right),
$$

so $g\left(z_{1}, z_{2} / z_{1}\right)=0$. If $d=\operatorname{degree}_{Z_{2}^{\prime}}(g)$, then

$$
0=z_{1}^{d} g\left(z_{1}, z_{2} / z_{1}\right)=g^{\prime}\left(z_{1}, z_{2}\right)
$$

which is not possible because $z_{1}, z_{2}$ are formally independent and $g^{\prime}\left(z_{1}, z_{2}\right)$ is a monic polynomial in $z_{2}$.

Remark 6 The above remarks 5 allow us to describe completely the discrete rank one valuations of $k\left(\left(X_{1}, X_{2}\right)\right) / k$ with our initial conditions.

(1) We start with $\left(X_{1}, X_{2}\right)$ and reduce it to the minimum value, obtaining a vector $\mathbf{z}=\left(z_{1}, z_{2}\right)$ whose components are formally independent over $k$ by remark 5.(3), and $k \llbracket z_{1}, z_{2} \rrbracket \supset k \llbracket X_{1}, X_{2} \rrbracket$. If the residue of $z_{2} / z_{1}$ belongs to $k$, we denote it by $\alpha$ and take the element $z_{2}^{\prime}=z_{2}-\alpha z_{1}$ whose value is strictly greater that $\widehat{v}\left(z_{1}\right)$. The vector $\left(z_{1}, z_{2}^{\prime}\right)$ has components again formally independent over $k$ and $k \llbracket z_{1}, z_{2}^{\prime} \rrbracket=k \llbracket z_{1}, z_{2} \rrbracket$.

(2) If $\widehat{v}\left(z_{2}^{\prime}\right)$ is a multiple of $\widehat{v}\left(z_{1}\right)$ we again reduce to the minimum value, which is $\widehat{v}\left(z_{1}\right)$, taking blowing-ups dividing by $z_{1}$. If, again, the residue is rational, we repeat and so on.

This process cannot be infinite because it would amount to an expansion of $z_{2}$ as a power series in $z_{1}$ with coefficients in $k$, which is not possible by formal independence (cf. remark 5.(3)). Therefore, the process stops, either because we arrive at an element whose value is not a multiple of $\widehat{v}\left(z_{1}\right)$ or we arrive at a residue which is transcendental over $k$. In the first case, we reduce again to minimum value getting an element of value strictly smaller than $\widehat{v}\left(z_{1}\right)$ and we start over from the beginning. In the second case, we stop.

This game of falling in the first case can be repeated only a finite number of times, because droppings of positive value can be only finitely many so, after a finite number of steps, we get a vector, renamed $\mathbf{z}=\left(z_{1}, z_{2}\right)$, in which the components have the same value and the residue $z_{2} / z_{1}$ is transcendental over $k$.

Moreover, and this is the most important fact, these components are formally independent over $k$ and $k \llbracket z_{1}, z_{2} \rrbracket \supset k \llbracket X_{1}, X_{2} \rrbracket$. Let $u_{2} \in \widehat{R}_{\widehat{v}}$ be the residue of $z_{2} / z_{1}$ and write $z_{2}=u_{2} z_{1}+z_{2}^{\prime}$ with $\widehat{v}\left(z_{2}^{\prime}\right)>\widehat{v}\left(z_{1}\right)$. Let us call $d$ the value $d=\widehat{v}\left(z_{1}\right)$. 
(3) The restriction $w$ of $\widehat{v}$ to $k \llbracket z_{1}, z_{2} \rrbracket$ is a monomial valuation. In fact, let $0 \neq f\left(z_{1}, z_{2}\right) \in k \llbracket z_{1}, z_{2} \rrbracket$ be a non-unit of order $r>0$ and let

$$
f\left(z_{1}, z_{2}\right)=\sum_{i \geq r} f_{i}\left(z_{1}, z_{2}\right)
$$

its decomposition into sum of forms; then, if we consider the inclusion $k \llbracket z_{1}, z_{2} \rrbracket \subset k\left(u_{2}\right) \llbracket z_{1} \rrbracket$, we can write

$$
f\left(z_{1}, z_{2}\right)=f\left(z_{1}, u_{2} z_{1}+z_{2}^{\prime}\right)=f_{r}\left(z_{1}, u_{2} z_{1}\right)+T=z_{1}^{r} f_{r}\left(1, u_{2}\right)+T
$$

where $f_{r}\left(1, u_{2}\right) \neq 0$ because $u_{2}$ is transcendental over $k$ and $T$ has value greater than $r w\left(z_{1}\right)=r d$ by proposition 3 . This proves our claim.

(4) However, (3) proves more. From it, we see that every non-unit power series of $k \llbracket z_{1}, z_{2} \rrbracket$ has a value which is a multiple of $d$. In particular, every non-unit power series in $k \llbracket X_{1}, X_{2} \rrbracket$ has the same property. Since the value group of $v$ is $\mathbb{Z}$, we must have $d=1$. We also see that the residue of every element in $K\left(\left(X_{1}, X_{2}\right)\right)$ is a rational function of $u_{2}$, hence $\Delta_{v}=k\left(u_{2}\right)$. Finally $v$ coincides with the composition of the (injective) ring homomorphism $\varphi: k \llbracket z_{1}, z_{2} \rrbracket \rightarrow k\left(u_{2}\right) \llbracket t \rrbracket$ given by $\varphi\left(z_{1}\right)=t, \varphi\left(z_{2}\right)=u_{2} t$ with the $t$-order function on $k\left(u_{2}\right) \llbracket t \rrbracket$.

These remarks prove the following theorem, due to Briales and Herrera [2, 3].

Theorem $5 A$ discrete rank one valuation $v$ of $k \llbracket X_{1}, X_{2} \rrbracket$ either is monomial or it can be reduced to a monomial one by a finite process consisting of blowing-ups and coordinate changes.

The rest of this section is devoted to listing all possible discrete rank one valuations of $k\left(\left(X_{1}, X_{2}, X_{3}\right)\right) / k$ whose ring contains $k \llbracket X_{1}, X_{2}, X_{3} \rrbracket$ and its center here is the maximal ideal. We fix one of them, $v$, and proceed.

Remark 7 (1) We start with the vector $\mathbf{X}=\left(X_{1}, X_{2}, X_{3}\right)$ and reduce it to its minimum value $d$; we get a vector $\mathbf{z}=\left(z_{1}, z_{2}, z_{3}\right)$ with $\widehat{v}\left(z_{i}\right)=d$, $i=1,2,3$. Let $\alpha_{i}$ be the residue of $z_{i} / z_{1}, i=2,3$; if both are elements of $k$, we take $z_{i}^{\prime}=z_{i}-\alpha_{i} z_{1}$ and the vector $\mathbf{z}^{\prime}=\left(z_{1}, z_{2}^{\prime}, z_{3}^{\prime}\right)$. If $\widehat{v}\left(z_{2}^{\prime}\right)$ and $\widehat{v}\left(z_{3}^{\prime}\right)$ are multiples of $d$, we reduce $\mathbf{z}^{\prime}$ to its minimum value by blowing-up dividing by $z_{1}$; let us rename $\mathbf{z}=\left(z_{1}, z_{2}, z_{3}\right)$ the output vector. If, again, the two residues belong to $k$ and the new values are multiple of $d$ we continue. The important point here is that, always, $k \llbracket \mathbf{X} \rrbracket \subset k \llbracket \mathbf{z} \rrbracket$.

(2) Is it possible to enter in an infinite process of this kind? In other words, is it possible to arrive to $k \llbracket \mathbf{X} \rrbracket \subset k \llbracket \mathbf{z} \rrbracket=k \llbracket z_{1} \rrbracket$ ? The answer is no. In fact, if affirmative, it will imply that all the $X_{i}$ belong to $k \llbracket z_{1} \rrbracket$ so, by Weierstrass preparation arguments, for each $i=2,3$ the $X_{i}$ will satisfy an equation of integral dependence over $k \llbracket X_{1} \rrbracket$, which is not the case. 
(3) Let us assume that, after a finite number of steps, we get a vector $\mathbf{z}$ such that, either $\widehat{v}\left(z_{2}\right)$ or $\widehat{v}\left(z_{3}\right)$ is not a multiple of $\widehat{v}\left(z_{1}\right)$. Then, reduction to minimum value will give us a vector, again renamed $\mathbf{z}$, such that the common value of its three components is $d^{\prime}<d$. If, again, we get residues in $k$ as in (1) and enter into a cycle as in there, we see by (2) that the cycle cannot be infinite.

(4) If, again, the minimum value drops and we enter into a cycle as in (1), and so on, we see that this process cannot be infinite, either. The reason is that a decreasing sequence of positive integers must stabilize.

(5) Therefore, after a finite number of steps, we must arrive at a vector $\mathbf{z}$ of equally valued components such that, after reordering if needed, the residue of $z_{2} / z_{1}$ is transcendental over $k$. In this process, we could have "lost" the component $z_{3}$. For instance, let us consider two variables $x, y$, the ring $k \llbracket x, y \rrbracket$ and, inside it, our starting ring $k \llbracket z_{1}, z_{2}, z_{3} \rrbracket$ with $z_{1}=y$, $z_{2}=x y, z_{3}=y f(x)$, where $f(x)$ is transcendental over $k(x)$. These $z_{1}, z_{2}, z_{3}$ are formally independent over $k$, as it is easy to see. Let us consider the monomial valuation $v$ of $k\left(\left(z_{1}, z_{2}, z_{3}\right)\right)$ such that $v\left(z_{1}\right)=1, v\left(z_{2}\right)=2$, $v\left(z_{3}\right)=1$; then $x=z_{2} / z_{1}$ has value $1, k \llbracket z_{1}, z_{2} / z_{1} \rrbracket=k \llbracket x, y \rrbracket$ and $z_{3} \in k \llbracket x, y \rrbracket$. Therefore, in all events, we could continue with the ring $k \llbracket z_{1}, z_{2} / z_{1} \rrbracket$ simply forgetting the $z_{3}$. In any case, deciding whether $z_{3}$ is lost or not could be the outcome of an infinite process. In fact, to see that $z_{3} \in k \llbracket z_{1}, z_{2} \rrbracket$ or not involves a (possibly infinite) process of blowing-ups.

(6) Let us assume that, in addition to the assumptions of remark 7.(5), $z_{3} \notin k \llbracket z_{1}, z_{2} \rrbracket$. Repeating a process similar to the one in (5), this time only with $z_{1}, z_{3}$, we could arrive either to a new fall of minimal value or to a residue transcendental over $k$. In case of fall of minimal value, we start everything from the beginning, and so on. It is evident that this process must be finite.

(7) The end of the history has two possibilities:

a) either a vector $\mathbf{z}=\left(z_{1}, z_{2}\right)$ of equally valued components such that the residue of $z_{2} / z_{1}$ is transcendental over $k$ and

$$
k \llbracket X_{1}, X_{2}, X_{3} \rrbracket \subset k \llbracket z_{1}, z_{2} \rrbracket,
$$

b) or not the above; then we have a vector $\mathbf{z}=\left(z_{1}, z_{2}, z_{3}\right)$ of equally positive valued components such that the residues of $z_{2} / z_{1}$ and $z_{3} / z_{1}$ are transcendental over $k$ (not necessarily algebraically independent over $k$ ) and

$$
k \llbracket X_{1}, X_{2}, X_{3} \rrbracket \subset k \llbracket z_{1}, z_{2}, z_{3} \rrbracket .
$$


Remark 8 In this note we consider the first case of the output in remark 7.(7). We have that $k \llbracket X_{1}, X_{2}, X_{3} \rrbracket \subset k \llbracket z_{1}, z_{2} \rrbracket$ and the residue $u$ of $z_{2} / z_{1}$ is transcendental over $k$. Let $d=\widehat{v}\left(z_{1}\right)=\widehat{v}\left(z_{2}\right)$ and write $z_{2}^{\prime}=z_{2}-u z_{1} \in \widehat{R}_{\widehat{v}}$ with $\widehat{v}\left(z_{2}^{\prime}\right)>d$. Let $\mathbf{Z}=\left(Z_{1}, Z_{2}\right)$ be a vector of indeterminates and $0 \neq f \in k \llbracket Z_{1}, Z_{2} \rrbracket$ be a power series of order $r>0$ and denote by $f_{i}\left(Z_{1}, Z_{2}\right)$ the forms of $f$; then

$$
f\left(z_{1}, z_{2}\right)=\sum_{i \geq r} f_{i}\left(z_{1}, u z_{1}+z_{2}^{\prime}\right)=f_{r}\left(z_{1}, u z_{1}\right)+T=z_{1}^{r} f_{r}(1, u)+T
$$

where $f_{r}(1, u) \neq 0$ by transcendence of $u$ and $T \in \widehat{R}_{\widehat{v}}, \widehat{v}(T)>r$. This shows that $\widehat{v}\left(f\left(z_{1}, z_{2}\right)\right)=r d$ and, since $k \llbracket X_{1}, X_{2}, X_{3} \rrbracket \subset k \llbracket z_{1}, z_{2} \rrbracket$ and the value group of $v$ is $\mathbb{Z}$, then it must be $d=1$. On the other hand, this also proves that the residue field of the restriction $w$ of $\widehat{v}$ to $k \llbracket z_{1}, z_{2} \rrbracket$ is $k(u)$, which is $\Delta_{v}$, is equal to $k(u)$. Of course, this restriction $w$ is a monomial valuation.

We can always write a concrete example of such a valuation by taking two formally independent variables $z_{1}, z_{2}$ a monomial valuation of $k \llbracket z_{1}, z_{2} \rrbracket / k$ and three formally independent power series in $k \llbracket z_{1}, z_{2} \rrbracket / k$.

Remark 9 In these notes we consider the second case of the output in remark 7.(7). We have that $k \llbracket X_{1}, X_{2}, X_{3} \rrbracket \subset k \llbracket z_{1}, z_{2}, z_{3} \rrbracket$ and the residues $u_{2,1}$ of $z_{2} / z_{1}$ and $u_{3,1}$ of $z_{3} / z_{1}$ are transcendental over $k$. In this case we need a little more preparation. Let $d=\widehat{v}\left(z_{1}\right)$.

(1) Now we initiate a process of coordinate changes and blowing-ups, similar to the one in remarks 7 , in search of an element of value strictly smaller than $d$, if it exists. We start with $\left(z_{1}, z_{2}\right)$, take the extension $L_{1}=$ $k\left(u_{2,1}\right)$ and the element $z_{2}^{\prime}=z_{2}-u_{2,1} z_{1}$. If $\widehat{v}\left(z_{2}^{\prime}\right)$ is a multiple of $\widehat{v}\left(z_{1}\right)$, then we equate values by taking blowing-ups dividing by $z_{1}$. Then we take again a suitable extension $L_{2}=L_{1}\left(u_{2, i_{2}}\right)$ and perform a coordinate change $z_{2}^{\prime \prime}=z_{2}-u_{2,1} z_{1}-u_{2, i_{2}} z_{1}^{i_{2}}$. If $\widehat{v}\left(z_{2}^{\prime \prime}\right)$ is a not a multiple of $\widehat{v}\left(z_{1}\right)$, then we take blowing-ups dividing by $z_{1}$ until we get an element of strictly smaller value. In this case, we restart everything (reduction to minimum value, coordinate changes, and so on, starting from a new three components vector), again over $k$ as the ground field.

For $\left(z_{1}, z_{2}\right)$, if we fall into an infinite process of values multiples of $\widehat{v}\left(z_{1}\right)$, we have a power series expansion

$$
z_{2}=\sum_{j \geq 1} u_{2, j} z_{1}^{j}, \quad u_{2, j} \in \Delta_{v} \backslash\{0\}
$$

and we act likewise with $\left(z_{1}, z_{3}\right)$. 
(2) This time, the end of the history is two power series expansions

$$
z_{i}=\sum_{j \geq 1} u_{i, j} z_{1}^{j}, \quad i=2,3 \quad u_{i, j} \in \Delta_{v} \backslash\{0\}
$$

where, of course, we have renamed the vector $\mathbf{z}$ and $u_{2,1}, u_{3,1}$ are transcendental over $k$. We denote by $L$ the extension of $k$ generated by all the $u_{i, j}$. If we write $z_{2}=u_{2,1} z_{1}^{\prime}$ then the map sending $z_{1}$ onto $z_{1}^{\prime}$ and $z_{i}$ onto $z_{i}$, $i=2,3$ is an $L$-automorphism of $L \llbracket \mathbf{z} \rrbracket$, so we may assume that $z_{2}=u z_{1}$, $u=u_{2,1}$. Therefore, we have

$$
\begin{aligned}
& z_{2}=u z_{1} \\
& z_{3}=\sum_{j \geq 1} u_{3, j} z_{1}^{j}
\end{aligned}
$$

so $L \llbracket z_{1}, z_{2}, z_{3} \rrbracket \subset L \llbracket z_{1} \rrbracket \subset \widehat{R}_{\widehat{v}}$ and $L \subset \Delta_{v}$.

(3) In the situation of (2), we consider the restriction $w$ of $\widehat{v}$ to $L \llbracket z_{1} \rrbracket$; then $w$ is necessarily the $z_{1}$-order function, so $\widehat{v}\left(z_{1}\right)=1$. Moreover, $\Delta_{v}=L$ and $\widehat{R}_{\widehat{v}}=L \llbracket z_{1} \rrbracket$.

So, in these remarks we have proved the following theorem:

Theorem 6 Let $v$ be a discrete rank one valuation of $k\left(\left(X_{1}, X_{2}, X_{3}\right)\right) / k$, whose ring contains $k \llbracket X_{1}, X_{2}, X_{3} \rrbracket$, its center here is the maximal ideal and the group of values is $\mathbb{Z}$. Then we have one of the following situations:

A) There exists a vector $\mathbf{z}=\left(z_{1}, z_{2}\right)$ of elements in $\widehat{\mathcal{M}}_{\widehat{v}}$ such that

$$
k \llbracket X_{1}, X_{2}, X_{3} \rrbracket \subset k \llbracket z_{1}, z_{2} \rrbracket, \quad v\left(z_{1}\right)=v\left(z_{2}\right)=1, \quad \Delta_{v}=k(u),
$$

where $u$ is the residue of $z_{2} / z_{1}$, and the restriction $w$ of $\widehat{v}$ to $k((\mathbf{z}))$ is a monomial valuation.

B) There exists a vector $\mathbf{z}=\left(z_{1}, z_{2}, z_{3}\right)$ of elements in $\widehat{\mathcal{M}}_{\widehat{v}}$ such that

$$
k \llbracket X_{1}, X_{2}, X_{3} \rrbracket \subset k \llbracket z_{1}, z_{2}, z_{3} \rrbracket, \quad v\left(z_{1}\right)=v\left(z_{2}\right)=v\left(z_{3}\right)=1,
$$

with $z_{2}=u z_{1}$ and $z_{3}=\sum_{j \geq 1} u_{3, j} z_{1}^{j}$, where $u$ and $u_{3,1}$ are transcendental residues over $k, \Delta_{v}=k\left(u,\left\{u_{3, j}\right\}_{j \geq 1}\right)$ and $\widehat{R}_{\widehat{v}}=\Delta_{v} \llbracket z_{1} \rrbracket$.

In both cases the vector $\mathbf{z}$ is explicitly obtained from $\mathbf{X}$ by a process consisting of blowing-ups and coordinate changes. 
Remark 10 In the situation of equation (3.1) the naturally arising problem is to study the field extension $k(u) \subset \Delta_{v}$. In this remark we deal with the case in which this extension is transcendental.

(1) By assumption, one of the coefficients $u_{3, j}$ must be transcendental over $k(u)$; let us call $u^{\prime}$ the first $u_{3, j}$ which is transcendental over $k(u)$, that is the one with the smallest possible $j=j_{0}$. If there are $u_{3, j} \neq 0$ with $j<j_{0}$ then we take the finite algebraic extension

$$
L=k(u)\left(\left\{u_{3, j}\right\}_{1 \leq j<j_{0}}\right) \supset k(u)
$$

and write

$$
\begin{aligned}
z_{3}^{\prime} & =\sum_{j=1}^{j_{0}-1} u_{3, j} z_{1}^{j} \\
z_{3}^{\prime \prime} & =u^{\prime} z_{1}^{j_{0}}+\sum_{j>j_{0}} u_{3, j} z_{1}^{j}
\end{aligned}
$$

We get the element $\left(1 / z_{1}^{j_{0}-1}\right) z_{3}^{\prime \prime}$ after a finite sequence of change of coordinates and blowing-ups dividing by $z_{1}$.

(2) Let us assume that $z_{3}^{\prime}$ does not exist, i.e., $z_{3}=u^{\prime} z_{1}^{j}+\cdots$; then the components of the vector $\mathbf{z}=\left(z_{1}, z_{2}, z_{3}\right)$ are formally independent over $k$ and $\Delta_{v}=k\left(u, u^{\prime}\right)$. To see this, let $\mathbf{Z}=\left(Z_{1}, Z_{2}, Z_{3}\right)$ be a vector of variables, $0 \neq f \in k \llbracket \mathbf{Z} \rrbracket$ be a non-unit of order $r$ and let $f_{i}(\mathbf{Z})$ be the form of degree $i$ of $f$; then

$$
f\left(z_{1}, z_{2}, z_{3}\right)=\sum_{i \geq r} f_{i}\left(z_{1}, z_{2}, z_{3}\right)=z_{1}^{r} f_{r}\left(1, u, u^{\prime}\right)+T
$$

where $T \in \widehat{\mathcal{M}}_{\widehat{v}}^{r+1}$ and $f_{r}\left(1, u, u^{\prime}\right) \neq 0$. This proves that $f\left(z_{1}, z_{2}, z_{3}\right) \neq 0$ and its residue is a polynomial in $u, u^{\prime}$, which implies our claim.

(3) Let us assume that $z_{3}^{\prime} \neq 0$ and let $L^{\prime} \supset L$ (cf. remark 10.(1)) be the minimal Galois extension of $k(u)$ containing $L$. Let

$$
P\left(z_{1}, Z_{3}\right)=\prod\left(Z_{3}-\left(z_{3}^{\prime}\right)^{(i)}\right)
$$

be the product taken over the different conjugates of $z_{3}^{\prime}$. Then $P\left(z_{1}, Z_{3}\right)$ is a polynomial in the indeterminate $Z_{3}$ with coefficients in $k(u)\left[z_{1}\right]$ such that $P\left(z_{1}, z_{3}^{\prime}\right)=0$ and, if $Q\left(z_{1}, Z_{3}\right) \in k(u)\left[z_{1}\right]$ is such that $Q\left(z_{1}, z_{3}^{\prime}\right)=0$ then $P\left(z_{1}, Z_{3}\right) \mid Q\left(z_{1}, Z_{3}\right)$. 
Now, let $0 \neq f\left(Z_{1}, Z_{2}, Z_{3}\right) \in k \llbracket Z_{1}, Z_{2}, Z_{3} \rrbracket$ be an irreducible non-unit such that

$$
\begin{aligned}
0 & =f\left(z_{1}, z_{2}, z_{3}\right)=f\left(z_{1}, u z_{1}, z_{3}^{\prime}+z_{3}^{\prime \prime}\right) \\
& =\sum_{i \geq 0} f_{i}\left(z_{1}, u z_{1}, z_{3}^{\prime}\right)\left(z_{3}^{\prime \prime}\right)^{i} .
\end{aligned}
$$

This implies that

$$
f_{i}\left(z_{1}, u z_{1}, z_{3}^{\prime}\right)=0, \quad \forall i \geq 0,
$$

so $P\left(z_{1}, Z_{3}\right) \mid f_{i}\left(z_{1}, u z_{1}, Z_{3}\right)$ for all $i$ by the Weierstrass preparation theorem. By irreducibility, $f\left(z_{1}, u z_{1}, Z_{3}\right)$ must be a unit factor of $P$. Hence, the initial form cannot be vanished by replacing $Z_{3}$ by $z_{3}^{\prime}+z_{3}^{\prime \prime}$. Therefore, $\left(z_{1}, z_{2}, z_{3}\right)$ are formally independent over $k$.

(4) It is obvious that there exist such valuations, for formally independent initial arguments. The composition of the following substitutions with the $t$-order functions give valuations, the first one being monomial and the second one being not:

$$
\begin{aligned}
& \varphi\left(X_{1}\right)=t, \quad \varphi\left(X_{2}\right)=u t, \varphi\left(X_{3}\right)=u^{\prime} t \\
& \varphi^{\prime}\left(X_{1}\right)=t, \quad \varphi^{\prime}\left(X_{2}\right)=u t, \quad \varphi^{\prime}\left(X_{3}\right)=(\sqrt{u}) t+u^{\prime} t^{2}
\end{aligned}
$$

Remark 11 We end with the case in which $\Delta_{v}$ is an algebraic extension of $k(u)$.

(1) In remark 7.(5) we saw how $\Delta_{v}$ can be a finite algebraic extension of $k(u)$ but not on the initial variables. It is also possible to get a non-trivial extension. Let us consider two variables $x, y$, the ring $k \llbracket x, y \rrbracket$ and, inside it, our starting ring $k \llbracket z_{1}, z_{2}, z_{3} \rrbracket$ with

$$
z_{1}=y^{2}, \quad z_{2}=x y^{2}, \quad z_{3}=y \exp (x),
$$

which are formally independent over $k$, as it is easy to see. Let us consider the monomial valuation $v$ of $k\left(\left(z_{1}, z_{2}, z_{3}\right)\right)$ such that $v\left(z_{1}\right)=2, v\left(z_{2}\right)=3$, $v\left(z_{3}\right)=1$; then $x=z_{2} / z_{1}$ has value $1, k \llbracket z_{1}, z_{2} / z_{1} \rrbracket=k \llbracket x, y^{2} \rrbracket$ and $z_{3}$ satifies $z_{3}^{2}=z_{1} \exp 2\left(z_{2} / z_{1}\right)$.

(2) It is also possible that the extension $\Delta_{v} \supset k(u)$ is infinite. In this case, $\left(z_{1}, z_{2}, z_{3}\right)$ are formally independent over $k$ as we can easily prove, reasoning by contradiction, using an Galois argument similar to the one in remark 10.(3). In other words, every countably generated algebraic extension of $k(u)$ can be realized as the residue field $\Delta_{v}$ of the valuation $v$. 


\section{Discrete rank two valuations in dimension two}

The principles of the techniques we have employed so far can be applied to other cases. We make a careful study of the discrete rank two valuations of $k \llbracket X_{1}, X_{2} \rrbracket$, just to illustrate the ideas. Let $v$ be a discrete rank two valuation of $K=k\left(\left(X_{1}, X_{2}\right)\right)$, finite over $R=k \llbracket X_{1}, X_{2} \rrbracket$ whose center at $R$ is the maximal ideal. We assume that the value group of $v$ is $\mathbb{Z}^{2}$, which is no restriction at all. This means, that there exist $z_{1}, z_{2} \in K$ such that $v\left(z_{1}\right)=(1,0), v\left(z_{2}\right)=(0,1)$.

Remark that the rank of any discrete valuation of $R$ is at most 2 because 2 vectors must generate a submodule of maximal rank of $\mathbb{Z}^{2}$. Remark, further, that the limitation of the rank by the dimension is by no means a consequence of the rather special starting situation. In fact, it is a determined by the Abhyankar-Zariski inequality (cf. [1]), valid for any local ring of finite dimension.

We observe that the valuation $v$ must be zero-dimensional. In fact, if the transcendence degree of $\Delta_{v} / k$ were positive, there could be a composite of the corresponding place with a non-trivial place of the residue field, which would be a valuation of higher rank. This is not possible by the limitation of the rank by the number of variables.

Remark 12 Let $u_{1}, u_{2}$ be variables, $F=k\left(\left(u_{2}\right)\right)\left(\left(u_{1}\right)\right), T=k\left(\left(u_{2}\right)\right) \llbracket u_{1} \rrbracket$ and let us define a special valuation in $F$ by giving its action on $T$.

(1) Any element in $T$ can be written as

$$
w=\sum_{i \geq 0} w_{i}\left(u_{2}\right) u_{1}^{i}, \quad w_{i}\left(u_{2}\right) \in k\left(\left(u_{2}\right)\right)
$$

We can write $w$ in another form as

$$
w=\sum_{(i, j) \in \mathbb{Z}_{0} \times \mathbb{Z}} a_{i j} u_{1}^{i} u_{2}^{j}, \quad a_{i, j} \in k
$$

and we will also write

$$
\mathcal{E}(w)=\left\{(i, j) \in \mathbb{Z}_{0} \times \mathbb{Z} \mid a_{i j} \neq 0\right\}
$$

Let us denote by $\nu_{u_{1}}, \nu_{u_{2}}$ the usual order of a power series in one variable (which can be negative). Assume $w \neq 0$; then it is very easy to see that

$$
\min _{\operatorname{lex}}(\mathcal{E})=\left(\nu_{u_{1}}(w), \nu_{u_{2}}\left(w_{\nu_{u_{1}}(w)}\left(u_{2}\right)\right)\right) .
$$


It also easy to see that the map $0 \neq w \mapsto \min _{\text {lex }}(\mathcal{E})$ defines a discrete rank two valuation $\hat{v}$ of $T$. Let us denote also by $\hat{v}$ the extension to $F$ of this valuation. The ring $R_{\hat{v}}$ consists of all the power series in $F$ with non-negative order in $u_{1}$ and positive or infinite order of the coefficient power series of $u_{1}^{0}=1$. Remark that $R_{\hat{v}} \subset T$ and $R_{\hat{v}} \neq T$; in fact all the terms in $T$ with order zero in $u_{1}$ and leading coefficient of negative order are out of $R_{\hat{v}}$.

(2) We can embed $R$ into $R_{\hat{v}}$ in such a way that $v$ extends uniquely to $\hat{v}$. This $R_{\hat{v}}$ plays the role of the completion in the rank one case.

(3) Assume that $v\left(X_{2}\right) \geq v\left(X_{1}\right)$; then the embedding of $R$ into $R_{\hat{v}}$ has a natural extension to an embedding of the blowing-up ring $k \llbracket X 1, X_{2} / X_{1} \rrbracket$ into $R_{\hat{v}}$. The proof is similar to the one in remark 5.(3).

Remark 13 (1) Let us assume that $v\left(X_{1}\right)$ and $v\left(X_{2}\right)$ are $\mathbb{Z}$-linearly independent; then each $L$-form with $L=\left\{v\left(X_{1}\right), v\left(X_{2}\right)\right\}$ is a monomial. In particular, $\left\{v\left(X_{1}\right), v\left(X_{2}\right)\right\}$ is a basis of $\mathbb{Z}^{2}$. Therefore, every such valuation of rank 2 of $k\left(\left(X_{1}, X_{2}\right)\right) / k$ is monomial and zero-dimensional.

(2) Let us assume that $v\left(X_{1}\right)$ and $v\left(X_{2}\right)$ are $\mathbb{Z}$-linearly dependent; then we apply a process of reduction to minimum value and a change of coordinates, as above. We repeat this again and again. After a possibly infinite sequence of blowing-ups and coordinate changes, we fall in a new vector $\left(y_{1}, y_{2}\right)$ in $\mathcal{M}_{\hat{v}}$ such that the values generate $\mathbb{Z}^{2}$. Then the valuation is monomial.

(3) We give an example of an infinite process. Let $v$ be the composition of the embedding

$$
\begin{aligned}
R & \longrightarrow R_{\hat{v}} \\
X_{1} & \longmapsto u_{2} \\
X_{2} & \longmapsto\left[\exp \left(u_{2}\right)-1\right]+\left[\exp \left(u_{1} / u_{2}\right)-1\right]
\end{aligned}
$$

with $\hat{v}$. The term $\left[\exp \left(u_{2}\right)-1\right]$ makes it necessary to take an infinite sequence of blowing-ups before finding a vector $\left(y_{1}, y_{2}\right)$ as before.

Remark 14 As a final remark, we point out that the process of reducing to the minimum value is, in all cases, similar to the exhaustion of the first characteristic exponent in the local resolution of the singularity of an analytically irreducible plane curve. 


\section{References}

[1] Abhyankar, S. S.: On the valuations centered in a local domain. Amer. J. Math. 78 (1956), 321-348.

[2] Briales, E. and Herrera, F. J.: Construcción explícita de las valoraciones de un anillo de series formales en dos variables. In Actas X Jornadas Hispano-Lusas (Murcia, 1985), II, 1-10. Universidad de Murcia, Murcia, 1985.

[3] Briales, E.: Constructive theory of valuations. Comm. Algebra 17 (1989), no. 5, 1161-1177.

[4] Vicente, J. L.: Aritmética en $\mathbb{Z}^{n}$. Revista de la Sociedad andaluza de profesores de matemáticas "THALES" (1986), no. 5, 8-32.

Recibido: 12 de abril de 2002

Revisado: 16 de julio de 2002

Francisco Javier Herrera Govantes
Departamento de Álgebra
Universidad de Sevilla
Apartado 116, 41080 Sevilla, Spain
jherrera@algebra.us.es
Miguel Ángel Olalla Acosta
Departamento de Álgebra
Universidad de Sevilla
Apartado 116, 41080 Sevilla, Spain
miguelolalla@algebra.us.es
José Luis Vicente Córdoba
Departamento de Álgebra
Universidad de Sevilla
jlvc@us.es

The authors are partially supported by FQM-218 (JdA). J. L. Vicente Córdoba and M. A. Olalla Acosta are partially supported by BFM 2001-3207 (MCyT). M. A. Olalla Acosta is also supported by HF2000-0044. 